\title{
Inequalities for Certain Hypergeometric Functions
}

\author{
By C. M. Joshi and J. P. Arya
}

\begin{abstract}
Theorems on two-sided inequalities for Gauss and Kummer's hypergeometric functions as given by Buschman have been improved. Complex analogues of the said inequalities have been developed and it is pointed out that a similar analysis gives extensions of Luke's, Flett's, and Carlson's theorems.
\end{abstract}

1. Let $F(\alpha)$ denote the hypergeometric function ${ }_{2} F_{1}(\alpha, \beta ; \gamma ; x)$. A contiguous relation for $F(\alpha)$, see, for instance, [6,2.8(28)] wherein $\alpha$ is replaced by $\alpha+n$ can be rewritten as

$$
F(\alpha+n+1)=A_{n} F(\alpha+n)+B_{n} F(\alpha+n-1),
$$

where

$$
A_{n}=\left(\frac{\beta x-\gamma}{\alpha+n}+2-x\right) /(1-x), \quad B_{n}=\left(\frac{\gamma}{\alpha+n}-1\right) /(1-x) .
$$

The recursion formula (1.1) enables us to represent $F(\alpha+n+1)$ by an $(n+2) \times$ $(n+2)$ determinant

$$
F(\alpha+n+1)=\operatorname{det}\left[\begin{array}{cccccc}
F(\alpha) & -F(\alpha-1) & & & & \\
B_{0} & A_{0} & -1 & & & \\
& B_{1} & A_{1} & -1 & & \\
& & & \ddots & & \\
& & & B_{n-1} & A_{n-1} & -1 \\
& & & & B_{n} & A_{n}
\end{array}\right] \text {. }
$$

The determinant (1.2) will have a strictly dominant diagonal provided that

$$
\begin{aligned}
& \text { (i) }|F(\alpha)|>|F(\alpha-1)|, \\
& \text { (ii) }\left|A_{k}\right|>\left|B_{k}\right|+1, \quad \text { for } 0<k<n \text {, } \\
& \text { (iii) }\left|A_{n}\right|>\left|B_{n}\right| .
\end{aligned}
$$

Assuming that $\alpha, \beta, \gamma$ and $x$ all are positive real numbers, an examination of power series representations of $F(\alpha)$ and $F(\alpha-1)$ with respect to $x$ shows that

$$
|F(\alpha)|>|F(\alpha-1)| \text { with } 0<x<1,
$$

Received October 21, 1980; revised May 12, 1981.

1980 Mathematics Subject Classification. Primary 33A30; Secondary 26D15, 15A15.

Key words and phrases. Gauss' hypergeometric function, Kummer's hypergeometric function, dominant diagonal matrix, complex parameters and arguments, inequalities. 
provided that

$$
\begin{aligned}
& \left|\frac{\alpha-1}{\alpha+n-1}\right| \ngtr 1 \quad \text { for } n \geqslant 1 \text { and } \\
& \left|\frac{\alpha-1}{\alpha+n-1}\right|<1 \text { for at least one value of } n .
\end{aligned}
$$

It therefore follows that 1.3 (i) holds for $\alpha \geqslant \frac{1}{2}$. Also, obviously 1.3(iii) will be valid under the same sets of conditions for which 1.3(ii) is valid. Now in order that 1.3(ii) may hold, in the first place, for $\gamma<\alpha$, it is sufficient that $\beta x>0$, which is obvious since $\beta$ and $x$ are both positive real numbers.

In the next place, consider the situation $\gamma>\alpha$. Let $\gamma>\alpha+k$ for some positive integer $k$. The inequality

$$
\left|A_{k}\right|>\left|B_{k}\right|+1
$$

will be satisfied for

$$
\beta>\beta x>\max \{\gamma, 2(\gamma-\alpha)\}>0
$$

Indeed this is so since in this case

$$
\left|A_{k}\right|=\left[\frac{\beta x-\gamma}{\alpha+k}+2-x\right] /(1-x), \quad\left|B_{k}\right|=\left(\frac{\gamma}{\alpha+k}-1\right) /(1-x) .
$$

If $\gamma>\alpha+n$, nothing remains to say, but if $\alpha<\gamma<\alpha+n$, there exists a nonnegative integer $k_{0}$ such that $\alpha+k_{0}<\gamma \leqslant \alpha+k_{0}+1$. Thus when $k>k_{0}$, (1.5) holds for $\beta x>0$, and when $k \leqslant k_{0}$, (1.5) holds under the conditions (1.6). Thus, the sufficient conditions under which 1.3(i)-1.3(iii) hold may be summarized as

$$
\alpha \geqslant \frac{1}{2}, \quad \gamma \leqslant \alpha \quad \text { or } \quad \beta>\beta x>\max \{\gamma, 2(\gamma-\alpha)\}>0 .
$$

Buschman [3] has claimed that (1.3) holds if all $\alpha, \beta, \gamma$ and $x$ are real and positive and satisfy the set of conditions $\alpha>1, \beta>\beta x>2 \gamma>0$. A closer examination clearly reveals that our conditions are much weaker than those given by Buschman and hence one can expect to get estimates in a wider range.

Thus under the conditions (1.7), by the theorem of G. B. Price [9] we have

$$
\begin{aligned}
A_{n}[F(\alpha)-|F(\alpha-1)|] \prod_{k=0}^{n-1} & \left(A_{k}-1\right)<{ }_{2} F_{1}(\alpha+n+1, \beta ; \gamma ; x) \\
& <A_{n}[F(\alpha)+|F(\alpha-1)|] \prod_{k=0}^{n-1}\left(A_{k}+1\right),
\end{aligned}
$$

where the absolute value symbols on $F(\alpha)$ and $A_{k}$ 's, $k=0, \ldots, n$, have been dropped because of our assumptions. Further, the absolute value symbol on $F(\alpha-1)$ can also be dropped by recourse to Erber's formula [5, (11)], which for real parameters and variables can be rewritten as

$$
\left|{ }_{2} F_{1}(a, b ; c ; z)\right| \leqslant{ }_{2} F_{1}(|a|,|b| ;|c| ;|z|) ; \quad|z|<1 .
$$

Consequently

$$
|F(\alpha-1)|={ }_{2} F_{1}(\alpha-1, \beta ; \gamma ; x) \mid \leqslant{ }_{2} F_{1}(|\alpha-1|, \beta ; \gamma ; x) .
$$


Hence converting products into gamma-functions, the result (1.8) along with (1.10) enables us to write the modified version of Buschman's Theorem 1 in the following form.

THEOREM 1. If $\alpha \geqslant \frac{1}{2}, \gamma \leqslant \alpha$ or $\beta>\beta x>\max \{\gamma, 2(\gamma-\alpha)\}>0$, then

$$
g(x) L<{ }_{2} F_{1}(\alpha+n+1, \beta ; \gamma ; x)<g(x) U,
$$

where

$$
\begin{gathered}
g(x)=(1-x)^{-n-1}(\beta x-\gamma+(2-x)(\alpha+n)) \Gamma(\alpha) / \Gamma(\alpha+n+1), \\
\text { (1.11) } U=[F(\alpha)+F(|\alpha-1|)](3-2 x)^{n} \Gamma\left(\frac{\beta x-\gamma}{3-2 x}+\alpha+n\right) / \Gamma\left(\frac{\beta x-\gamma}{3-2 x}+\alpha\right), \\
L=[F(\alpha)-F(|\alpha-1|)] \Gamma(\beta x-\gamma+\alpha+n) / \Gamma(\beta x-\gamma+\alpha) .
\end{gathered}
$$

It is observed here that for $\alpha>1, F(|\alpha-1|)=F(\alpha-1)$, and therefore $U$ and $L$ of (1.11) correspond to those of Theorem 1 of Buschman [3]. Further, by using the bounds for the determinant (1.2) as given by Brenner [1], we have

THEOREM 2. If $\alpha \geqslant \frac{1}{2}, \gamma \leqslant \alpha$ or $\beta>\beta x>\max \{\gamma, 2(\gamma-\alpha)\}>0$, then

$$
L^{\prime}<{ }_{2} F_{1}(\alpha+n+1, \beta ; \gamma ; x)<U^{\prime} \text {, }
$$

where

$$
\begin{aligned}
L^{\prime} & =\left(\frac{F^{2}(\alpha)-F^{2}(\alpha-1)}{F(\alpha)}\right) \prod_{k=0}^{n-1}\left(A_{k}-\frac{\left|B_{k}\right|+1}{A_{k}}\right), \\
U^{\prime} & =A_{n}\left(\frac{F^{2}(\alpha)+F^{2}(\alpha-1)}{F(\alpha)}\right) \prod_{k=0}^{n-1}\left(A_{k}+\frac{\left|B_{k}\right|+1}{A_{k}}\right) .
\end{aligned}
$$

It should be noted that the absolute value symbol on $B_{k}$ 's can be dropped when $\gamma>\alpha+n$. Note also that whereas Theorem 2 gives improved lower and upper bounds for ${ }_{2} F_{1}(\alpha+n+1, \beta ; \gamma ; x)$ over Theorem 1 , Theorem 1 is more suitable in applications because of its simplicity.

Improvements over Theorems 1 and 2, though in a restrictive domain, may further be obtained in the light of the suggestions made by Srivastava and Brenner [10] by writing the determinant (1.2) in the alternate form

(1.13) $F(\alpha+n+1)=\operatorname{det}\left[\begin{array}{ccccc}F(\alpha) & -F(\alpha-1) \vee B_{0} & & & \\ \vee B & A_{0} & & -\vee B_{1} & \\ & & \ddots & & \\ & & \vee B_{n-1} & A_{n-1} & -\vee B_{n} \\ & & & \vee B_{n} & A_{n}\end{array}\right]$.

Thus, for example, the inequality corresponding to Theorem 1 may be stated as follows:

THEOREM 3. If $\alpha>\frac{1}{2}$, and either $\alpha \beta>\beta \gamma>\alpha \beta x>\gamma>0$, or $\alpha(1-x)>\gamma-\alpha$ $>0, \beta x>\max \{\gamma, 2(\gamma-\alpha)\}>0$, then

$$
L^{\prime \prime}<{ }_{2} F_{1}(\alpha+n+1 ; \beta ; \gamma ; x)<U^{\prime \prime},
$$


where

$$
\begin{aligned}
& L^{\prime \prime}=A_{n}\left[F(\alpha)-F(|\alpha-1|)\left|\vee B_{0}\right|\right] \prod_{k=0}^{n-1}\left(A_{k}-\left|\vee B_{k+1}\right|\right), \\
& U^{\prime \prime}=A_{n}\left[F(\alpha)+F(|\alpha-1|)\left|\vee B_{0}\right|\right] \prod_{k=0}^{n-1}\left(A_{k}+\left|\vee B_{k+1}\right|\right) .
\end{aligned}
$$

If $1<\alpha<\gamma$, the ${ }_{2} F_{1}$ 's in the bounds of the above listed theorems can further be approximated by application of Luke's [8, 4.21, 4.23], Carlson's [4] or Flett's [7] theorems to obtain inequalities in terms of parameters and variables.

Proceeding as before, an improved version of Theorem 2 of Buschman [3] can be stated as

THEOREM 4. If $\alpha \geqslant \frac{1}{2}, \alpha \geqslant \gamma>0$ or $x>\max \{\gamma, 2(\gamma-\alpha)\}>0$, then

$$
h(x) B<{ }_{1} F_{1}(\alpha+n+1 ; \gamma ; x)<h(x) A \text {, }
$$

where

$$
\begin{gathered}
h(x)=(x-\gamma+2(\alpha+n)) \Gamma(\alpha) / \Gamma(\alpha+n+1), \\
A=\left[{ }_{1} F_{1}(\alpha ; \gamma ; x)+{ }_{1} F_{1}(|\alpha-1| ; \gamma ; x)\right] 3^{n} \Gamma\left(\frac{x-\gamma}{3}+\alpha+n\right) / \Gamma\left(\frac{x-\gamma}{3}+\alpha\right), \\
B=\left[{ }_{1} F_{1}(\alpha ; \gamma ; x)-{ }_{1} F_{1}(|\alpha-1| ; \gamma ; x)\right] \Gamma(x-\gamma+\alpha+n) / \Gamma(x-\gamma+\alpha) .
\end{gathered}
$$

Also, by the same analysis, it is found that Theorem 3 of Buschman, which gives bounds for the confluent hypergeometric function $\Psi$, is valid in a larger domain $2 c-1>a>0, x>0$.

2. The Case of Complex Parameters and Variables. Erber [5] observed that for $n>0$,

$$
\left|(\alpha)_{n}\right| \leqslant(|\alpha|)_{n}, \quad\left|(\alpha)_{n}\right| \geqslant(\cos (\theta / 2))^{n-1}(|\alpha|)_{n}, \quad \theta=\arg \alpha,|\theta|<\pi,
$$

and used these to obtain

$$
\left.\right|_{2} F_{1}(\alpha, \beta ; \gamma ; z) \mid \leqslant \cos (\theta / 2)_{2} F_{1}(|\alpha|,|\beta| ;|\gamma| ;|z| \sec \theta / 2),
$$

where $\theta=\arg \gamma,|\theta|<\pi$, and $|z|<\cos (\theta / 2)$. From (2.1) we can also have

$$
\left.\right|_{p} F_{q}\left(\alpha_{p} ; \beta_{q} ; z\right) \mid \leqslant \Pi \cos \left(\theta_{q} / 2\right)_{p} F_{q}\left(\left|\alpha_{p}\right| ;\left|\beta_{q}\right| ;|z| \Pi \sec \left(\theta_{q} / 2\right)\right),
$$

where $\theta_{q}=\arg \left(\beta_{q}\right),\left|\theta_{q}\right|<\pi,|z|<\Pi \cos \left(\theta_{q} / 2\right)$, and as usual $\Pi$ stands for the product symbol. If $p \leqslant q$, the condition $|z|<\Pi \cos \left(\theta_{q} / 2\right)$ in (2.3) can be dropped.

With the help of (2.2) and the triangle inequality $|\alpha+n|<|\alpha|+n, n$ being any nonnegative integer, extensions of Theorems 1,3 , and 4 for complex parameters and arguments can be obtained. For reasons of brevity we shall however state only the extension of Theorem 1.

THEOREM 5. If $a, b, c$, and $z$ are complex numbers and $\theta=\arg c,|\theta|<\pi$, $|z|<\cos (\theta / 2)$, then

$$
\left|{ }_{2} F_{1}(a+n+1, b ; c ; z)\right|<\cos (\theta / 2) U \cdot g(z),
$$


where

$$
\begin{aligned}
g(z)= & \frac{[1-|z| \sec (\theta / 2)]^{-n-1}}{(|a|)_{n+1}}[|b z| \sec (\theta / 2)-|c|+(2-|z| \sec (\theta / 2))(|a|+n)], \\
U= & \left\{{ }_{2} F_{1}(|a|,|b| ;|c| ;|z| \sec (\theta / 2))+{ }_{2} F_{1}(|| a|-1|,|b| ;|c| ;|z| \sec (\theta / 2))\right\} \\
& \cdot(3-2|z| \sec (\theta / 2))^{n}((|b z| \sec (\theta / 2)-|c|) /(3-2|z| \sec (\theta / 2))+|a|)_{n},
\end{aligned}
$$

provided

$$
|a| \geqslant \frac{1}{2}, \quad|c| \leqslant|a| \text { or }|b|>|b z| \sec (\theta / 2)>\max \{|c|, 2(|c|-|a|)\}>0 .
$$

In the sequel, complex analogues of inequalities of Luke $[8,4.21,4.23,5.6,5.8]$ and those of Flett [7] and Carlson [4] could also be given similarly.

Acknowledgements. Thanks are due to the referee for his very valuable suggestions.

Department of Mathematics

University of Jodhpur

Jodhpur (Rajasthan), India

Department of Mathematics

D.A.V. College

Muzaffarnagar (U.P.), India

1. J. L. Brenner, "A bound for a determinant with dominant main diagonal," Proc. Amer. Math. Soc., v. 5, 1954, pp. 631-634.

2. J. L. BRENNER, "Bounds for classical polynomials derivable by matrix methods," Proc. Amer. Math. Soc., v. 30, 1971, pp. 353-362.

3. R. G. Buschman, "Inequalities for hypergeometric functions," Math. Comp., v. 30, 1976, pp. 303-305.

4. B. C. Carlson, "Some inequalities for hypergeometric functions," Proc. Amer. Math. Soc., v. 17, 1966, pp. 32-39.

5. T. ERBER, "Inequalities for hypergeometric functions," Arch. Rational Mech. Anal., v. 4, 1959/1960, pp. 341-351.

6. A. ERdélyi et AL., Higher Transcendental Functions, Vol. I, McGraw-Hill, New York, 1953.

7. T. M. FLetr, "Some inequalities for a hypergeometric integral," Proc. Edinburgh Math. Soc. (2), v. 18, 1972, pp. 31-34.

8. Y. L. LuKE, "Inequalities for generalized hypergeometric functions," J. Approx. Theory, v. 5, 1972, pp. 41-65.

9. G. B. Price, "Bounds for determinants with dominant principal diagonal," Proc. Amer. Math. Soc., v. 2, 1951, pp. 497-502.

10. H. M. SRivastava \& J. L. Brenner, "Bounds for Jacobi and related polynomials derivable by matrix methods," J. Approx. Theory, v. 12, 1974, pp. 372-377. 